\title{
A Study on $N_{\theta}$-Quasi-Cauchy Sequences
}

\author{
Hüseyin Çakalli ${ }^{1}$ and Huseyin Kaplan ${ }^{2}$ \\ ${ }^{1}$ Maltepe University, Department of Mathematics, Faculty of Arts and Science, Marmara Eğitim Köyü, Maltepe, \\ 34857 Istanbul, Turkey \\ ${ }^{2}$ Department of Mathematics, Niğde University, Faculty of Science and Letters, 051100 Niğde, Turkey
}

Correspondence should be addressed to Hüseyin Çakalli; hcakalli@maltepe.edu.tr

Received 14 November 2012; Revised 24 January 2013; Accepted 15 February 2013

Academic Editor: Ziemowit Popowicz

Copyright (C) 2013 H. Cakalli and H. Kaplan. This is an open access article distributed under the Creative Commons Attribution License, which permits unrestricted use, distribution, and reproduction in any medium, provided the original work is properly cited.

Recently, the concept of $N_{\theta}$-ward continuity was introduced and studied. In this paper, we prove that the uniform limit of $N_{\theta}$-ward continuous functions is $N_{\theta}$-ward continuous, and the set of all $N_{\theta}$-ward continuous functions is a closed subset of the set of all continuous functions. We also obtain that a real function $f$ defined on an interval $E$ is uniformly continuous if and only if $\left(f\left(\alpha_{k}\right)\right)$ is $N_{\theta}$-quasi-Cauchy whenever $\left(\alpha_{k}\right)$ is a quasi-Cauchy sequence of points in $E$.

\section{Introduction}

The concept of continuity and any concept involving continuity play a very important role not only in pure mathematics but also in other branches of sciences involving mathematics especially in computer science, information theory, and biological science.

A real function $f$ is continuous if and only if it preserves convergent sequences. A subset $E$ of $\mathbf{R}$, the set of real numbers, is compact if any sequence of points in $E$ has a convergent subsequence whose limit is in $E$. Using the idea of continuity of a real function and the idea of compactness in terms of sequences, many kinds of continuities and compactness were introduced and investigated, not all but some of them we recall in the following: slowly oscillating continuity, slowly oscillating compactness [1], quasislowly oscillating continuity, quasi-slowly oscillating compactness [2], $\Delta$-quasi-slowly oscillating continuity, $\Delta$-quasislowly oscillating compactness [3-5], ward continuity, ward compactness [6,7], $\delta$-ward continuity, $\delta$-ward compactness [8], statistical ward continuity, and lacunary statistical ward continuity $[9,10]$.

In [11], the notion of $N_{\theta}$ convergence was introduced, and studied by Freedman et al. Using the main idea for continuity and compactness given above the concepts of $N_{\theta^{-}}$ ward compactness of a subset $E$ of $\mathbf{R}$ and $N_{\theta}$-ward continuity of a real function are introduced and investigated recently in [12].

The purpose of this paper is to continue the investigation given in [12] and obtain further interesting results on $N_{\theta^{-}}$ ward continuity.

\section{Preliminaries}

Boldface letters $\boldsymbol{\alpha}, \mathbf{x}, \mathbf{y}, \mathbf{z}, \ldots$ will be used for sequences $\boldsymbol{\alpha}=$ $\left(\alpha_{k}\right), \boldsymbol{\alpha}=\left(x_{n}\right), \mathbf{y}=\left(y_{n}\right), \mathbf{z}=\left(z_{n}\right), \ldots$ of points in the set of real numbers $\mathbf{R}$ for the sake of abbreviation. Sums of the form $\sum_{k_{r-1}+1}^{k_{r}}\left|\alpha_{k}\right|$ frequently occur and will often be written for convenience as $\sum_{k \in I_{r}}\left|\alpha_{k}\right|$.

The concept of a Cauchy sequence involves far more than that the distance between successive terms is tending to zero. Nevertheless, sequences which satisfy this weaker property are interesting in their own right. A sequence $\left(\alpha_{n}\right)$ of points in $\mathbf{R}$ is quasi-Cauchy if $\left(\Delta \alpha_{n}\right)$ is a null sequence where $\Delta \alpha_{n}=$ $\alpha_{n+1}-\alpha_{n}$. These sequences were named as quasi-Cauchy by Burton and Coleman [13, page 328], while they were called as forward convergent to 0 sequences in [7, page 226]. 
A sequence $\left(\alpha_{k}\right)$ of points in $\mathbf{R}$ is called $N_{\theta}$-convergent to an element $L$ of $\mathbf{R}$ if

$$
\lim _{r \rightarrow \infty} \frac{1}{h_{r}} \sum_{k \in I_{r}}\left|\alpha_{k}-L\right|=0,
$$

where $I_{r}=\left(k_{r-1}, k_{r}\right]$ and $\theta=\left(k_{r}\right)$ is a lacunary sequence, that is, an increasing sequence of positive integers such that $k_{0}=0$ and $h_{r}: k_{r}-k_{r-1} \rightarrow \infty$. The intervals determined by $\theta$ are denoted by $I_{r}=\left(k_{r-1}, k_{r}\right]$, and the ratio $k_{r} / k_{r-1}$ is abbreviated by $q_{r}$. A sequence $\left(\alpha_{n}\right)$ of points in $\mathbf{R}$ is called $N_{\theta}$-quasi-Cauchy if $\left(\Delta \alpha_{n}\right)$ is $N_{\theta}$-convergent to 0 . A function defined on a subset $A$ of $\mathbf{R}$ is called $N_{\theta}$-ward continuous if it preserves $N_{\theta}$-quasi-Cauchy sequences, that is, $\left(f\left(\alpha_{k}\right)\right)$ is an $N_{\theta}$-quasi-Cauchy sequence whenever $\left(\alpha_{k}\right)$ is. $\left|\Delta N_{\theta}^{0}\right|$ will denote the set of $N_{\theta}$-quasi-Cauchy sequences of points in R. Any subsequence of a Cauchy sequence is Cauchy. The analogous property fails for $N_{\theta}$-quasi-Cauchy sequences. A counterexample for the case is the sequence $\left(a_{n}\right)=(\sqrt{n})$ with the subsequence $\left(a_{n^{2}}\right)=(n)$.

A sequence $\left(\alpha_{n}\right)$ of points in $\mathbf{R}$ is slowly oscillating [14, Definition 2, page 947] if

$$
\lim _{\lambda \rightarrow 1^{+}} \varlimsup_{n} \max _{n+1 \leq k \leq[\lambda n]}\left|\alpha_{k}-\alpha_{n}\right|=0,
$$

where $[\lambda n]$ denotes the integer part of $\lambda n$ (see also [14]).

An ideal $I$ is a family of subsets of positive integers $\mathbf{N}$ which is closed under taking finite unions and subsets of its elements. A sequence $\left(\alpha_{n}\right)$ of real numbers is said to be ideal convergent to a real number $L$, if for each $\varepsilon>0$ the set $\left\{n:\left|\alpha_{n}-L\right| \geq \varepsilon\right\}$ belongs to $I$. Ideal ward compactness of a subset of $\mathbf{R}$ and ideal ward continuity of a real function were recently introduced by Çakalli and Hazarika in [15].

\section{Results}

Any quasi-Cauchy sequence is $N_{\theta}$-quasi-Cauchy, so any slowly oscillating sequence is $N_{\theta}$-quasi-Cauchy, and so any Cauchy sequence is. A sequence $\boldsymbol{\alpha}=\left(\alpha_{k}\right)$ is called Cesaro summable to a real number $L$ if $\lim _{n \rightarrow \infty}(1 / n) \sum_{i=1}^{n} \alpha_{i}=L$. This is denoted by $C_{1}-\lim \alpha_{k}=L$, and the set of all Cesaro sequences is denoted by $\sigma_{1}$. We call a sequence $\boldsymbol{\alpha}=\left(\alpha_{k}\right)$ Cesaro quasi-Cauchy if $C_{1}-\lim \Delta \alpha_{k}=0$. The set of all Cesaro quasi-Cauchy sequences is denoted by $\Delta \sigma_{1}^{0}$. A sequence $\boldsymbol{\alpha}=$ $\left(\alpha_{k}\right)$ is called strongly Cesaro summable to a real number $L$ if $\lim _{n \rightarrow \infty}(1 / n) \sum_{i=1}^{n}\left|\alpha_{i}-L\right|=0$. This is denoted by $\left|C_{1}\right|-\lim \alpha_{k}=L$. The set of all strongly Cesaro summable sequences is denoted by $\left|\sigma_{1}\right|$. We call a sequence $\boldsymbol{\alpha}=\left(\alpha_{k}\right)$ strongly Cesaro quasi-Cauchy if $\left|C_{1}\right|-\lim \Delta \alpha_{k}=0$. The set of all strongly Cesaro quasi-Cauchy sequences is denoted by $\left|\Delta \sigma_{1}^{0}\right|$. The following inclusions are satisfied: $\left|\sigma_{1}\right| \subset \sigma_{1}$ and $\left|\Delta \sigma_{1}^{0}\right| \subset \Delta \sigma_{1}^{0}$.

Using a similar idea to that of [11], one can easily find out the following inclusion properties between the set of strongly Cesaro quasi-Cauchy sequences and the set of $N_{\theta^{-}}$ quasi-Cauchy sequences (see also [16]).

(i) $\left|\Delta N_{\theta}^{0}\right| \subset\left|\Delta \sigma_{1}^{0}\right|$ if and only if lim $\sup q_{r}<\infty$ for any lacunary sequence $\theta$. (ii) $\left|\Delta \sigma_{1}^{0}\right| \subset\left|\Delta N_{\theta}^{0}\right|$ if and only if lim inf $q_{r}>1$ for any lacunary sequence $\theta$.

Combining these facts, for any lacunary sequence $\theta$, we have the following:

(iii) $\left|\Delta N_{\theta}^{0}\right|=\left|\Delta \sigma_{1}^{0}\right|$ if and only if $1<\liminf q_{r} \leq$ $\lim \sup q_{r}<\infty$;

(iv) $\left|\Delta N_{\theta}^{0}\right|=\left|\Delta \sigma_{1}^{0}\right|$ if and only if $\left|\sigma_{1}\right|=N_{\theta}^{0}$.

In the sequel, we will always assume that $\lim _{\inf } q_{r}>1$.

We observe that $N_{\theta}$-summability is a kind of strong $A$ summability where $A=\left(a_{r k}\right)$ is a regular matrix generated by the lacunary sequence $\theta=\left(k_{r}\right)$ as follows:

$$
a_{r k}=\frac{1}{h_{r}} \quad \text { if } k \in I_{r}, a_{r k}=0 \text { otherwise. }
$$

On the other hand, we see that $N_{\theta}$-ward continuity cannot be given as a strong $A$-continuity by any kind of regular summability matrix (related to continuity for strong matrix methods see [17]).

As far as ideal continuity is considered, we note that any $N_{\theta}$-ward continuous function is ideal continuous; furthermore any $N_{\theta}$ continuous function is ideal continuous for an admissible ideal.

Theorem 1. If a function $f$ is uniformly continuous on a subset E of $\mathbf{R}$, then, $\left(f\left(\alpha_{k}\right)\right)$ is $N_{\theta}$-quasi-Cauchy whenever $\left(\alpha_{k}\right)$ is a quasi-Cauchy sequence of points in $E$.

Proof. Let $E$ be a subset of $\mathbf{R}$, and let $f$ be a uniformly continuous function on $E$. Take any quasi-Cauchy sequence $\left(\alpha_{k}\right)$ of points in $E$, and let $\varepsilon$ be any positive real number. By uniform continuity of $f$, there exists a $\delta>0$ such that $|f(\alpha)-f(\beta)|<\varepsilon$ whenever $|\alpha-\beta|<\delta$ and $\alpha, \beta \in E$. Since $\left(\alpha_{k}\right)$ is a quasi-Cauchy sequence, there exists a positive integer $k_{0}$ such that $\left|\alpha_{k+1}-\alpha_{k}\right|<\delta$ for $k \geq k_{0}$. Hence,

$$
\frac{1}{h_{r}} \sum_{k \in I_{r}}\left|f\left(\alpha_{k+1}\right)-f\left(\alpha_{k}\right)\right|<\frac{1}{h_{r}}\left(k_{r}-k_{r-1}\right) \varepsilon=\varepsilon,
$$

for $r \geq k_{0}$. Thus, $\left(f\left(\alpha_{k}\right)\right)$ is an $N_{\theta}$-quasi-Cauchy sequence. This completes the proof of the theorem.

We have much more below for a real function $f$ defined on an interval that $f$ is uniformly continuous if and only if $\left(f\left(\alpha_{k}\right)\right)$ is $N_{\theta}$-quasi-Cauchy whenever $\left(\alpha_{k}\right)$ is a quasi-Cauchy sequence of points in $E$. First, we give the following lemma.

Lemma 2. If $\left(\xi_{n}, \eta_{n}\right)$ is a sequence of ordered pairs of points in an interval such that $\lim _{n \rightarrow \infty}\left|\xi_{n}-\eta_{n}\right|=0$, then, there exists an $N_{\theta}$-quasi-Cauchy sequence $\left(\alpha_{n}\right)$ with the property that for any positive integer $i$ there exists a positive integer $j$ such that $\left(\xi_{i}, \eta_{i}\right)=\left(\alpha_{j-1}, \alpha_{j}\right)$.

Proof. Although the following proof is similar to that of [13], we give it for completeness. For each positive integer $k$, we can 
fix $z_{0}^{k}, z_{1}^{k}, \ldots, z_{n_{k}}^{k}$ in $E$ with $z_{0}^{k}=\eta_{k}, z_{n_{k}}^{k}=\xi_{k+1}$, and $\left|z_{i}^{k}-z_{i-1}^{k}\right|<$ $1 / k$ for $1 \leq i \leq n_{k}$. Now write

$$
\begin{aligned}
& \left(\xi_{1}, \eta_{1}, z_{1}^{1}, \ldots, z_{n_{1}-1}^{1}, \xi_{2}, \eta_{2}, z_{1}^{2}, \ldots, z_{n_{2}-1}^{2}, \xi_{3}, \eta_{3}, \ldots,\right. \\
& \left.\xi_{k}, \eta_{k}, z_{1}^{k}, \ldots, z_{n_{k-1}}^{k}, \xi_{k+1}, \eta_{k+1}, \ldots\right)
\end{aligned}
$$

Then denoting this sequence by $\left(\alpha_{n}\right)$, we obtain that for any positive integer $i$ there exists a positive integer $j$ such that $\left(\xi_{i}, \eta_{i}\right)=\left(\alpha_{j-1}, \alpha_{j}\right)$. The sequence constructed is a quasiCauchy sequence, and it is an $N_{\theta}$-quasi-Cauchy sequence, since any quasi-Cauchy sequence is an $N_{\theta}$-quasi-Cauchy sequence. This completes the proof of the lemma.

Theorem 3. If a function $f$ defined on an interval $E$ is $N_{\theta^{-}}$ ward continuous, then, it is uniformly continuous.

Proof. Suppose that $f$ is not uniformly continuous on $E$. Then, there is an $\varepsilon_{0}>0$ such that for any $\delta>0$ there exist $x, y \in E$ with $|x-y|<\delta$ but $|f(x)-f(y)| \geq \varepsilon_{0}$. For every integer $n \geq 1$ fix $\xi_{n}, \eta_{n} \in E$ with $\left|\xi_{n}-\eta_{n}\right|<1 / n$ and $\left|f\left(\xi_{n}\right)-f\left(\eta_{n}\right)\right| \geq \varepsilon_{0}$. By the lemma, there exists an $N_{\theta^{-}}$ quasi-Cauchy sequence $\left(\alpha_{i}\right)$ such that for any integer $i \geq 1$ there exists a $j$ with $\xi_{i}=\alpha_{j}$ and $\eta_{i}=\alpha_{j+1}$. This implies that $\mid f\left(\alpha_{j+1}\right)-f\left(\alpha_{j)} \mid \geq \varepsilon_{0}\right.$; hence, $\left(f\left(\alpha_{i}\right)\right)$ is not $N_{\theta}$-quasi-Cauchy. Thus, $f$ does not preserve $N_{\theta}$-quasi-Cauchy sequences. This completes the proof of the theorem.

Observing that the sequence, constructed in the proof of the preceding theorem, is also a quasi-Cauchy sequence, we obtain that a real function $f$ defined on an interval $E$ is uniformly continuous if $\left(f\left(\alpha_{k}\right)\right)$ is $N_{\theta}$-quasi-Cauchy whenever $\left(\alpha_{k}\right)$ is a quasi-Cauchy sequence of points in $E$. Combining this with Theorem 1, we have that a real function $f$ defined on an interval $E$ is uniformly continuous if and only if $\left(f\left(\alpha_{k}\right)\right)$ is $N_{\theta}$-quasi-Cauchy whenever $\left(\alpha_{k}\right)$ is a quasiCauchy sequence of points in $E$.

Corollary 4. If a function defined on an interval is $N_{\theta}$-ward continuous, then, it is ward continuous.

Proof. The proof follows from Theorem 3 and [7, Theorem 6] so it is omitted.

Corollary 5. If a function defined on an interval is $N_{\theta}$-ward continuous, then, it is slowly oscillating continuous.

Proof. The proof follows from Theorem 3 and [7, Theorem 5] so it is omitted.

It is a well-known result that uniform limit of a sequence of continuous functions is continuous. This is also true in case of $N_{\theta}$-ward continuity; that is, uniform limit of a sequence of $N_{\theta}$-ward continuous functions is $N_{\theta}$-ward continuous.

Theorem 6. If $\left(f_{n}\right)$ is a sequence of $N_{\theta}$-ward continuous functions on a subset $E$ of $\mathbf{R}$ and $\left(f_{n}\right)$ is uniformly convergent to a function $f$, then, $f$ is $N_{\theta}$-ward continuous on $E$.

Proof. Let $\left(\alpha_{k}\right)$ be any $N_{\theta}$-quasi-Cauchy sequence of points in $E$, and let $\varepsilon$ be any positive real number. By uniform convergence of $\left(f_{n}\right)$, there exists an $n_{1} \in \mathbf{N}$ such that $\mid f(\alpha)-$ $f_{k}(\alpha) \mid<\varepsilon / 3$ for $n \geq n_{1}$ and every $\alpha \in E$. Hence,

$$
\frac{1}{h_{r}} \sum_{k \in I_{r}}\left|f(\alpha)-f_{k}(\alpha)\right|<\frac{1}{h_{r}}\left(k_{r}-k_{r-1}\right) \frac{\varepsilon}{3}=\frac{\varepsilon}{3},
$$

for $r \geq n_{1}$ and every $\alpha \in E$. As $f_{n_{1}}$ is $N_{\theta}$-ward continuous on $E$, there exists an $n_{2} \in \mathbf{N}$ such that for $r \geq n_{2}$

$$
\frac{1}{h_{r}} \sum_{k \in I_{r}}\left|f_{n_{1}}\left(\alpha_{k+1}\right)-f_{n_{1}}\left(\alpha_{k}\right)\right|<\frac{\varepsilon}{3} .
$$

Now write $n_{0}=\max \left\{n_{1}, n_{2}\right\}$. Thus for $r \geq n_{0}$, we have

$$
\begin{aligned}
& \frac{1}{h_{r}} \sum_{k \in I_{r}}\left|f\left(\alpha_{k+1}\right)-f\left(\alpha_{k}\right)\right| \\
& \leq \frac{1}{h_{r}} \sum_{k \in I_{r}}\left|f\left(\alpha_{k+1}\right)-f_{n_{1}}\left(\alpha_{k+1}\right)\right| \\
&+\frac{1}{h_{r}} \sum_{k \in I_{r}}\left|f_{n_{1}}\left(\alpha_{k+1}\right)-f_{n_{1}}\left(\alpha_{k}\right)\right| \\
&+\frac{1}{h_{r}} \sum_{k \in I_{r}}\left|f_{n_{1}}\left(\alpha_{k}\right)-f\left(\alpha_{k}\right)\right| \\
&< \frac{\varepsilon}{3}+\frac{\varepsilon}{3}+\frac{\varepsilon}{3}=\varepsilon
\end{aligned}
$$

Hence,

$$
\lim _{r \rightarrow \infty} \frac{1}{h_{r}} \sum_{k \in I_{r}}\left|f\left(\alpha_{k+1}\right)-f\left(\alpha_{k}\right)\right|=0 .
$$

Thus, $f$ preserves $N_{\theta}$-quasi-Cauchy sequences. This completes the proof of the theorem.

Theorem 7. The set of all $N_{\theta}$-ward continuous functions on a subset $E$ of $\mathbf{R}$ is a closed subset of the set of all continuous functions on $E$, that is, $\overline{\Delta N_{\theta} W C(E)}=\Delta N_{\theta} W C(E)$, where $\Delta N_{\theta} W C(E)$ is the set of all $N_{\theta}$-ward continuous functions on $E$ and $\overline{\triangle N_{\theta} W C(E)}$ denotes the set of all cluster points of $\Delta N_{\theta} W C(E)$.

Proof. Let $f$ be any element in the closure of $\Delta N_{\theta} W C(E)$. Then, there exists a sequence $\left(f_{n}\right)$ of points in $\Delta N_{\theta} W C(E)$ such that $\lim _{k \rightarrow \infty} f_{k}=f$. To show that $f$ is $N_{\theta}$-ward continuous, take any $N_{\theta}$-quasi-Cauchy sequence $\left(\alpha_{k}\right)$ of points in $E$. Let $\varepsilon>0$. Since $\left(f_{n}\right)$ converges to $f$, there exists an $n_{1} \in \mathbf{N}$ such that $\left|f\left(\alpha_{k}\right)-f_{n_{1}}\left(\alpha_{k}\right)\right|<\varepsilon / 3$ for all $k \in \mathbf{N}$. Hence,

$$
\frac{1}{h_{r}} \sum_{k \in I_{r}}\left|f\left(\alpha_{k}\right)-f_{n_{1}}\left(\alpha_{k}\right)\right|<\frac{1}{h_{r}}\left(k_{r}-k_{r-1}\right) \frac{\varepsilon}{3}=\frac{\varepsilon}{3},
$$

for $r \geq n_{1}$. As $f_{n_{1}}$ is $N_{\theta}$-ward continuous on $E$, there exists a positive integer $n_{2} \in \mathbf{N}$ such that $r \geq n_{2}$ implies that

$$
\frac{1}{h_{r}} \sum_{k \in I_{r}}\left|f_{n_{1}}\left(\alpha_{k+1}\right)-f_{n_{1}}\left(\alpha_{k}\right)\right|<\frac{\varepsilon}{3} .
$$


Now write $n_{0}=\max \left\{n_{1}, n_{2}\right\}$. Thus for $r \geq n_{0}$, we have

$$
\begin{aligned}
\frac{1}{h_{r}} \sum_{k \in I_{r}} & \left|f\left(\alpha_{k+1}\right)-f\left(\alpha_{k}\right)\right| \\
\leq & \frac{1}{h_{r}} \sum_{k \in I_{r}}\left|f\left(\alpha_{k+1}\right)-f_{n_{1}}\left(\alpha_{k+1}\right)\right| \\
& +\frac{1}{h_{r}} \sum_{k \in I_{r}}\left|f_{n_{1}}\left(\alpha_{k+1}\right)-f_{n_{1}}\left(\alpha_{k}\right)\right| \\
& +\frac{1}{h_{r}} \sum_{k \in I_{r}}\left|f_{n_{1}}\left(\alpha_{k}\right)-f\left(\alpha_{k}\right)\right| \\
< & \frac{\varepsilon}{3}+\frac{\varepsilon}{3}+\frac{\varepsilon}{3}=\varepsilon ;
\end{aligned}
$$

Hence,

$$
\lim _{r \rightarrow \infty} \frac{1}{h_{r}} \sum_{k \in I_{r}}\left|f\left(\alpha_{k+1}\right)-f\left(\alpha_{k}\right)\right|=0 .
$$

Thus, $f$ preserves $N_{\theta}$-quasi-Cauchy sequences. This completes the proof of the theorem.

Corollary 8. The set of all $N_{\theta}$-ward continuous functions on a subset $E$ of $\mathbf{R}$ is a complete subspace of the space of all continuous functions on $E$.

Proof. The proof follows from the preceding theorem.

\section{Conclusion}

In this paper, new results concerning $N_{\theta}$-ward continuity are obtained namely; a real function $f$ defined on an interval $E$ is uniformly continuous if and only if $\left(f\left(\alpha_{k}\right)\right)$ is $N_{\theta}$-quasiCauchy whenever $\left(\alpha_{k}\right)$ is a quasi-Cauchy sequence of points in $E$, the uniform limit of $N_{\theta}$-ward continuous functions is $N_{\theta}$-ward continuous, and the set of all $N_{\theta}$-ward continuous functions is a closed subset of the set of all continuous functions. We also prove that if a function $f$ is uniformly continuous on a subset $E$ of $\mathbf{R}$, then, $\left(f\left(\alpha_{k}\right)\right)$ is $N_{\theta}$-quasiCauchy whenever $\left(\alpha_{k}\right)$ is a quasi-Cauchy sequence of points in $E$.

As a further study one can find out if Theorem 3 is valid when the set $E$ is replaced by a $G$-sequentially connected subset of $\mathbf{R}$ for a regular sequential method $G$ [18]. For another further study, we suggest to investigate the present work for the fuzzy case. However, due to the change in settings, the definitions and methods of proofs will not always be analogous to those of the present work (see [19] for the definitions in the fuzzy setting). One can introduce and give an investigation of $N_{\theta}$-quasi-Cauchy sequences in cone normed spaces (see [20] for basic concepts in cone normed spaces).

\section{References}

[1] H. Çakalli, "Slowly oscillating continuity," Abstract and Applied Analysis, vol. 2008, Article ID 485706, 5 pages, 2008.
[2] M. Dik and I. Çanak, "New types of continuities," Abstract and Applied Analysis, vol. 2010, Article ID 258980, 6 pages, 2010.

[3] H. Çakalli, I. Çanak, and M. Dik, " $\Delta$-quasi-slowly oscillating continuity," Applied Mathematics and Computation, vol. 216, no. 10, pp. 2865-2868, 2010.

[4] H. Çakalli, "New kinds of continuities," Computers \& Mathematics with Applications, vol. 61, no. 4, pp. 960-965, 2011.

[5] H. Çakalli, "On $\Delta$-quasi-slowly oscillating sequences," Computers \& Mathematics with Applications, vol. 62, no. 9, pp. 35673574, 2011.

[6] H. Çakalli, "Forward compactness," in Proceedings of the Conference on Summability and Applications, Shawnee State University, November 2009.

[7] H. Çakalli, "Forward continuity," Journal of Computational Analysis and Applications, vol. 13, no. 2, pp. 225-230, 2011.

[8] H. Çakalli, “ $\delta$-quasi-Cauchy sequences," Mathematical and Computer Modelling, vol. 53, no. 1-2, pp. 397-401, 2011.

[9] H. Çakalli, "Statistical ward continuity," Applied Mathematics Letters, vol. 24, no. 10, pp. 1724-1728, 2011.

[10] H. Çakalli, "Statistical quasi-Cauchy sequences," Mathematical and Computer Modelling, vol. 54, no. 5-6, pp. 1620-1624, 2011.

[11] A. R. Freedman, J. J. Sember, and M. Raphael, "Some Cesarotype summability spaces," Proceedings of the London Mathematical Society, vol. 37, no. 3, pp. 508-520, 1978.

[12] H. Çakalli, " $N_{\theta}$-ward continuity," Abstract and Applied Analysis, vol. 2012, Article ID 680456, 8 pages, 2012.

[13] D. Burton and J. Coleman, "Quasi-Cauchy sequences," American Mathematical Monthly, vol. 117, no. 4, pp. 328-333, 2010.

[14] F. Dik, M. Dik, and I. Çanak, "Applications of subsequential Tauberian theory to classical Tauberian theory," Applied Mathematics Letters, vol. 20, no. 8, pp. 946-950, 2007.

[15] H. Çakalli and B. Hazarika, "Ideal quasi-Cauchy sequences," Journal of Inequalities and Applications, vol. 2012, article 234, 2012.

[16] H. Çakalli, "Lacunary statistical convergence in topological groups," Indian Journal of Pure and Applied Mathematics, vol. 26, no. 2, pp. 113-119, 1995.

[17] J. Connor and K.-G. Grosse-Erdmann, "Sequential definitions of continuity for real functions," The Rocky Mountain Journal of Mathematics, vol. 33, no. 1, pp. 93-121, 2003.

[18] H. Çakalli, "Sequential definitions of connectedness," Applied Mathematics Letters, vol. 25, no. 3, pp. 461-465, 2012.

[19] H. Çakalli and P. Das, "Fuzzy compactness via summability," Applied Mathematics Letters, vol. 22, no. 11, pp. 1665-1669, 2009.

[20] A. Sonmez and H. Cakalli, "Cone normed spaces and weighted means," Mathematical and Computer Modelling, vol. 52, no. 910, pp. 1660-1666, 2010. 


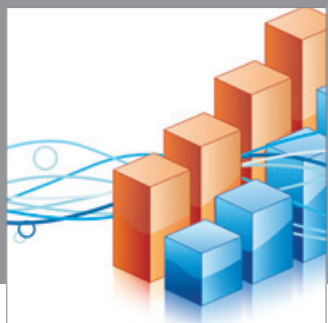

Advances in

Operations Research

mansans

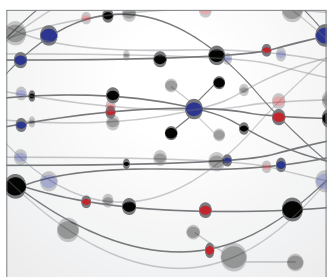

The Scientific World Journal
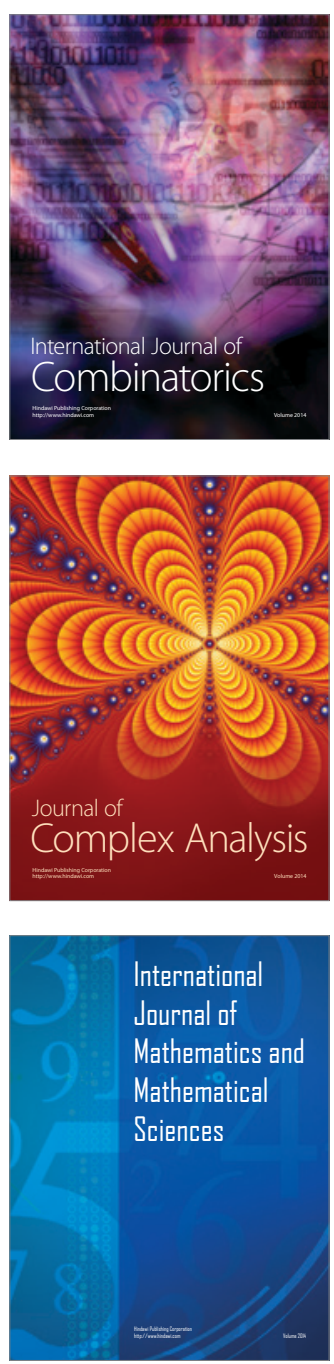
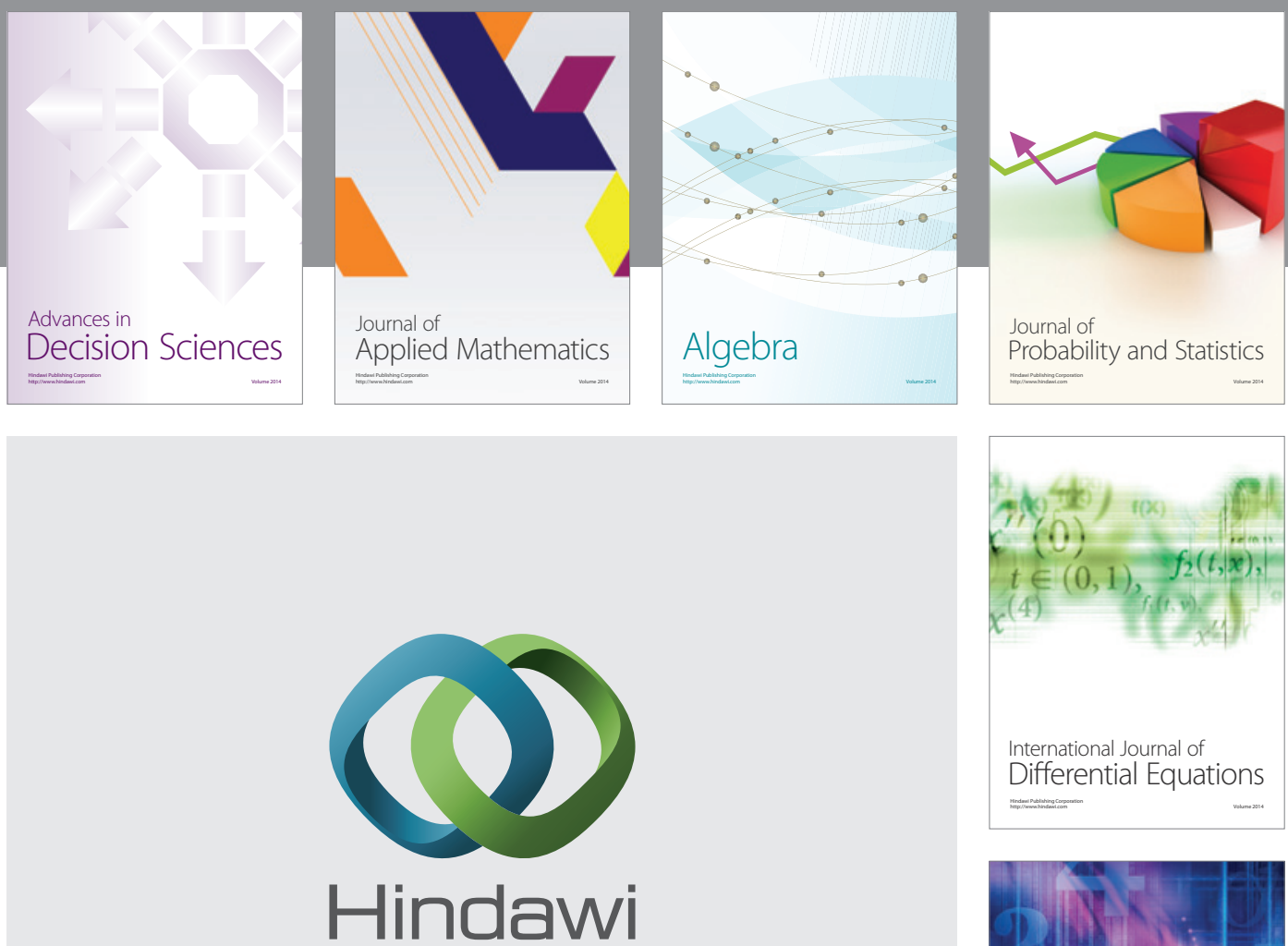

Submit your manuscripts at http://www.hindawi.com
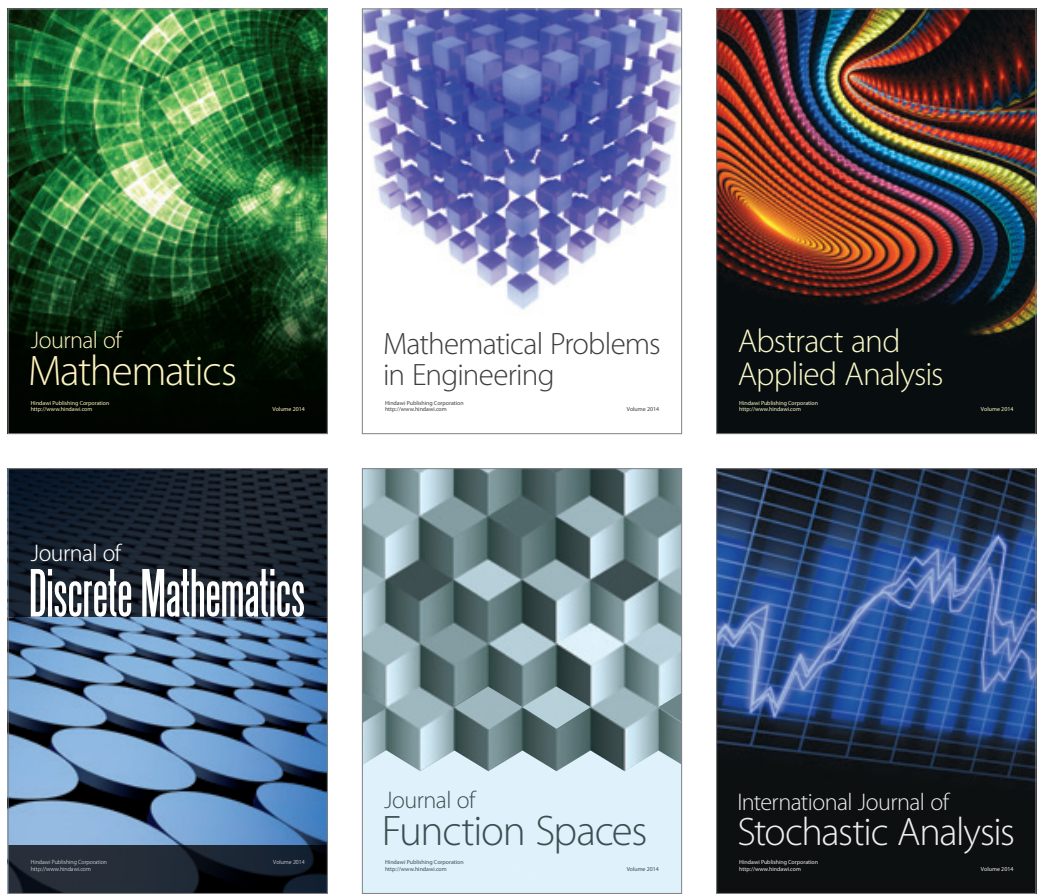

Journal of

Function Spaces

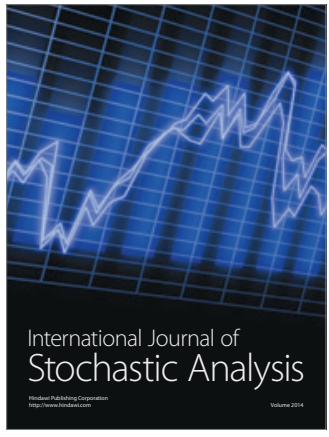

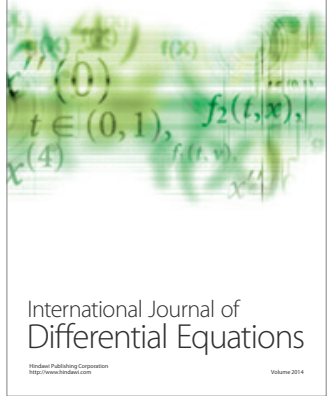
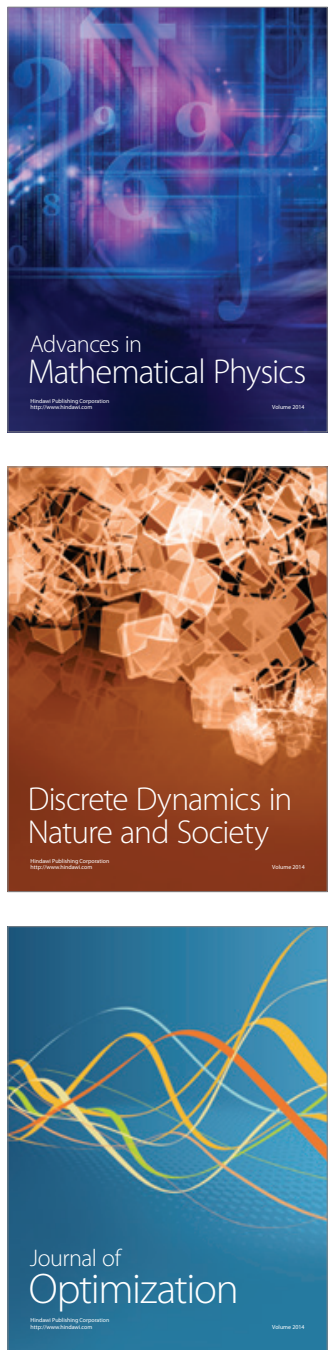